\title{
The DNA methylation profile of non-coding RNAs improves prognosis prediction for pancreatic adenocarcinoma
}

Jie Zhang ${ }^{1}$, Keqing Shi' ${ }^{2}$ Weiguo Huang ${ }^{1}$, Wanqing Weng' ${ }^{1}$, Zhongjing Zhang ${ }^{1}$, Yangyang Guo ${ }^{1}$, Tuo Deng ${ }^{1}$, Yukai Xiang ${ }^{1}$, Xiaofeng $\mathrm{Ni}^{1}$, Bicheng Chen ${ }^{1}$ and Mengtao Zhou ${ }^{1,2^{*}}$

\begin{abstract}
Background: Compelling lines of evidence indicate that DNA methylation of non-coding RNAs (ncRNAs) plays critical roles in various tumour progression. In addition, the differential methylation of ncRNAs can predict prognosis of patients. However, little is known about the clear relationship between DNA methylation profile of ncRNAs and the prognosis of pancreatic adenocarcinoma (PAC) patients.

Methods: The data of DNA methylation, RNA-seq, miRNA-seq and clinical features of PAC patients were collected from TCGA database. The DNA methylation profile was obtained using the Infinium HumanMethylation450 BeadChip array. LASSO regression was performed to construct two methylation-based classifiers. The risk score of methylationbased classifiers was calculated for each patient, and the accuracy of the classifiers in predicting overall survival (OS) was examined by ROC curve analysis. In addition, Cox regression models were utilized to assess whether clinical variables and the classifiers were independent prognostic factors for OS. The targets of miRNA and the genes coexpressed with IncRNA were identified with DIANA microT-CDS and the Multi-Experiment Matrix (MEM), respectively. Moreover, DAVID Bioinformatics Resources were applied to analyse the functional enrichment of these targets and co-expressed genes.
\end{abstract}

Results: A total of $4004 \mathrm{CpG}$ sites of miRNA and 11,259 CpG sites of IncRNA were screened. Among these CpG sites, $8 \mathrm{CpG}$ sites of miRNA and $7 \mathrm{CpG}$ sites of IncRNA were found with regression coefficients. By multiplying the sum of methylation degrees of the selected CpGs with these coefficients, two methylation-based classifiers were constructed. The classifiers have shown good performance in predicting the survival rate of PAC patients at varying follow-up times. Interestingly, both of these two classifiers were predominant and independent factors for OS. Furthermore, functional enrichment analysis demonstrated that aberrantly methylated miRNAs and IncRNAs are related to calcium ion transmembrane transport and MAPK, Ras and calcium signalling pathways.

Conclusion: In the present study, we identified two methylation-based classifiers of ncRNA associated with OS in PAC patients through a comprehensive analysis of miRNA and IncRNA profiles. We are the first group to demonstrate a relationship between the aberrant DNA methylation of ncRNAs and the prognosis of PAC, and this relationship would contribute to individualized PAC therapy.

Keywords: DNA methylation, Pancreatic adenocarcinoma, miRNA, IncRNA, Classifier, Prognosis

\footnotetext{
*Correspondence: zhoumengtao@wmu.edu.cn

${ }^{1}$ Key Laboratory of Diagnosis and Treatment of Severe Hepato-Pancreatic

Diseases of Zhejiang Province, The First Affiliated Hospital, Wenzhou

Medical University, Wenzhou 325015, Zhejiang Province, People's

Republic of China

Full list of author information is available at the end of the article
}

(c) The Author(s) 2019. This article is distributed under the terms of the Creative Commons Attribution 4.0 International License (http://creativecommons.org/licenses/by/4.0/), which permits unrestricted use, distribution, and reproduction in any medium, provided you give appropriate credit to the original author(s) and the source, provide a link to the Creative Commons license, and indicate if changes were made. The Creative Commons Public Domain Dedication waiver (http://creativecommons.org/ publicdomain/zero/1.0/) applies to the data made available in this article, unless otherwise stated. 


\section{Background}

Pancreatic adenocarcinoma (PAC) is one of the most prevalent type of highly lethal malignancy in the digestive system. It is the fourth most dominant cancer-associated death, with a 5-year survival rate less than 5\% [1]. Ttreatment of PAC, including surgical resection, radiotherapy and chemotherapy, has been improved in the recent years [2]. However, even with these disease management options, the overall survival (OS) rates of PAC patients are still far from satisfactory. The poor effectiveness of treatment therapies results from the high aggressiveness of PAC, its resistance to chemotherapy drugs, and a lack of early diagnosis [3]. Therefore, to reduce mortality and improve the management of PAC patients, identification of prognosis biomarkers during the early stage of PAC is extremely important.

Non-coding RNAs (ncRNAs) have been widely found in complex eukaryotic organisms, and have gradually emerged as a new research focus because of their unique biological roles in tumour progression [4]. Although they cannot be translated into proteins, ncRNAs play critical roles in chromosomal modification, transcriptional interference, post-transcriptional modification and translational regulation via epigenetic modifications in human cells [5]. Emerging evidence has revealed that ncRNAs with dysregulated expression profiles are involved in the pathogenesis of multiple cancers, including colorectal cancer [6], breast cancer [7], as well as gastric cancer [8]. Furthermore, epigenetic modifications, especially DNA methylation, have been determined to play pivotal roles in the regulation of protein-encoding genes, miRNAs and lncRNAs [9]. Zhang et al. [10] demonstrated that the tumour suppressor microRNA-596 (miR-596) was silenced by hypermethylation at its relevant promoter in gastric cancer but not in normal tissues. Furthermore, the miR-596 expression of gastric cancer cell lines was reduced, accompanied by an increment of miR-596 methylation, which is consistent with previous study. miR-770 has also been reported to be significantly decreased in gastric cardia adenocarcinoma (GCA) as a result of the hypermethylation of the lncRNA coded by MEG3. Additionally, the aberrant methylated CpG sites of MEG3 and dysregulated miR-770 content were closely related to a poor prognosis for patients [11]. These studies indicated that abnormal DNA methylation could be a vital biomarker in the pathophysiology and evaluation of cancers. Hence, we hypothesize that the methylation of ncRNAs can predict the prognosis of PAC.

To date, the prognostic values assessed by the methylation of ncRNAs and the association between miRNAs and lncRNAs in PAC remain poorly elucidated. In our present study, differential methylation patterns of miRNA and lncRNA were analysed in PAC using the
485,577 sites identified by the HumanMethylation 450 BeadChip, which is annotated by The Cancer Genome Atlas (TCGA). Using the Least Absolute Shrinkage and Selection Operator (LASSO) regression model, we successfully developed miRNA and lncRNA methylationbased classifiers to assess OS of patients. Functional enrichment analysis of differently methylated ncRNAs was performed to analyse the target genes and the association between miRNAs and lncRNAs.

\section{Materials and methods \\ Patient datasets}

The data of DNA methylation, RNA-seq, miRNA-seq and clinical features in PAC patients were from TCGA (https ://portal.gdc.cancer.gov/). DNA methylation profiles was determined via the Infinium HumanMethylation450 BeadChip. Additionally, RNA-seq and miRNA-seq were processed on the Illumina HiSeq RNA-seq and miRNAseq platforms, respectively. The lncRNA annotation file was obtained from GENCODE (https://www.gencodegen es.org/). According to the publication guidelines, all of the datasets used in this study were publicly available.

\section{CpG sites of miRNAs and IncRNAs}

The extent of methylation of a $\mathrm{CpG}$ site is characterized using a beta-value, ranging from 0 (unmethylated) to 1 (completely methylated). We used the "minfi" package in R software for normalization of methylation beta-values. On the basis of the annotations by the TCGA, CpG sites within $2 \mathrm{~kb}$ upstream of the transcriptional start site (TSS) of lncRNA or miRNA were chosen from the 485,577 sites of the HumanMethylation450 BeadChip. Using the "minf" package in R software, miRNA and lncRNA sites that were differentially methylated between PAC tissues and normal adjacent tissues were identified, and q-values $<0.05$ were considered statistically significant. To calculate the different beta-values of CpG sites between PAC tissues and normal adjacent tissues, a $t$ test was performed and beta-values differences greater than 0.1 were considered significant. Afterwards, correlation analysis was applied to identify these sites to determine where methylation levels were negatively correlated with the expression of miRNA or lncRNA. The results were adopted when the P-values was less than 0.05 .

\section{Methylation-based classifiers for overall survival}

The LASSO regression model [12] was used to identify the most accurate predictive CpGs. For example, if there were two different methylated CpGs sites in parallel, LASSO would automatically filter out the secondary related one and assign the selected CpGs site a value, which equals the regression coefficient in the classifier formulas. Meanwhile, the beta value in 
the formulas represents the methylation degree of the selected CpG site of miRNA or lncRNA. Herein, with the unique method of LASSO by the "glmnet" package in $\mathrm{R}$ software, we made the sum of the methylation degrees of the selected CpGs weighted by the coefficients and developed a linear model under high dimensional conditions with the least regression coefficients to maximize the prediction accuracy in PAC prognosis.

\section{Predictive and prognostic analysis \\ of the methylation-based classifiers}

Patient risk scores were determined based on the methylation-based classifiers, and time-dependent receiver operating characteristic (tdROC) curve analysis was applied to evaluate the predictive accuracy of classifiers for OS based on the risk score. To assess whether clinical variables and the classifiers were independent prognostic factors for OS, we utilized univariate and multivariate Cox regression models to identify significant prognostic predictors associated with OS. The prognostic evaluation of clinical variables and classifiers was also performed with tdROC analysis using the "timeROC" package of $\mathrm{R}$ software. The predictive or prognostic accuracy was indicated by the area under the curve (AUC) of tdROC. We used the median of the classifier risk score as a cut-off point to categorized the patients into two groups, including high-risk groups and low-risk groups. The survival estimation of patients were analysed with the Kaplan-Meier method. In addition, P-values $<0.05$ were considered statistically significant.

\section{miRNA target genes, IncRNA co-expression genes}

\section{and functional enrichment analysis}

The DIANA microT-CDS tool (http://diana.imis.athen a-innovation.gr/) were applied to predict the target genes of miRNA. The co-expression genes of lncRNA were identified with the Multi-Experiment Matrix (MEM) [13]. MEM has a large collections of microarray data sets. Using simultaneous statistical significance estimation, MEM applies rank aggregation to merge information from different data sets into a single global ordering. We used MEM to perform a gene expression similarity search across many data sets (https://biit.cs.ut.ee/mem/). We then performed functional enrichment analysis of the target genes and co-expression genes using DAVID Bioinformatics Resources (https://david.ncifcrf.gov/). It was considered statistically significant when false discovery rates (FDRs) of enrichment terms were less than 0.05 . The major enrichment terms were visualized using the "ggplot2" package in R software.

\section{Results}

Characteristics of patient datasets

We studied 184 PAC patients, and collected both DNA methylation data and survival data from the TCGA. Table 1 illustrated the main clinical characteristics of the 184 PAC patients. The methylation data comprised 194 samples, including 184 PAC samples and 10 samples of normal adjacent tissues for corresponding PAC patients. The miRNA-seq data included 181 samples, and the RNA-seq data included 182 samples.

\section{Differential methylation of $\mathrm{CpG}$ sites and methylation-based classifier}

A total of 4004 CpG sites of miRNA and 11,259 CpG sites of lncRNA located within $2 \mathrm{~kb}$ upstream of the miRNA or lncRNA TSS (excluding the CpG sites on the $\mathrm{X}$ and $\mathrm{Y}$ chromosomes) were screened according to the HumanMethylation450 BeadChip annotations in the TCGA. In total, $511 \mathrm{CpG}$ sites of miRNA and $1441 \mathrm{CpG}$ sites of lncRNA with different methylation patterns between PAC and normal adjacent tissues were screened and analysed using the "minfi" package in R software; $273 \mathrm{CpG}$ sites of miRNA and 809 CpG sites of lncRNA had betavalue differences greater than 0.1. Among these $\mathrm{CpG}$ sites, the methylation levels of $86 \mathrm{CpG}$ sites of miRNA and $348 \mathrm{CpG}$ sites of lncRNA were negatively related to the expression levels of miRNA or lncRNA.

To develop a methylation-based classifier to predict the prognosis of PAC, a LASSO regression model was carried out by the methylation data of $86 \mathrm{CpG}$ sites of miRNA and 348 CpG sites of lncRNA, respectively. 8

Table 1 Clinical characteristics of pancreatic adenocarcinoma

\begin{tabular}{lr}
\hline Clinicopathological variables & $\mathbf{n}=\mathbf{1 8 4}$ \\
\hline Age (years) & \\
$<60$ & $57(31.0 \%)$ \\
$\geq 60$ & $127(69.0 \%)$ \\
Gender & \\
Male & $102(55.4 \%)$ \\
Female & $82(44.6 \%)$ \\
Tumor grade & \\
G1+ G2 & $129(70.1 \%)$ \\
G3+ G4 & $53(28.8 \%)$ \\
Pathological tumor size & \\
T1 + T2 & $31(16.8 \%)$ \\
T3+T4 & $151(82.1 \%)$ \\
Pathological stage & \\
I+II & $172(93.5 \%)$ \\
III + IV & $9(4.9 \%)$ \\
Recurrence & $83(45.1 \%)$ \\
Death & $99(53.8 \%)$ \\
\hline
\end{tabular}


CpG sites of miRNA and 7 CpG sites of lncRNA were found with regression coefficients (Fig. 1a, b, d, e). The risk score formulas for methylation-based classifiers were established as follows: methylation-based classifier for $\operatorname{miRNA}=0.057 \times$ beta_cg03003746 $+0.033 \times$ beta c g03593550+0.081 × beta_cg05376374 $-0.155 \times$ beta_cg13766329 $+0.099 \times$ beta_cg19267861 $0.214 \times$ beta_cg $21236500-0.072 \times$ beta_cg22358580+ $0.469 \times$ beta_cg 23651812 , methylation-based classifier for lncRNA $=0.075 \times$ beta_cg06722407 $-0.266 \times$ beta_cg06892907 + $0.046 \times$ beta_cg $12918457+$ $0.010 \times$ beta_cg $20892260+0.283 \times$ beta_cg23651812 + $0.071 \times$ beta_cg $23883696+0.040 \times$ beta_cg25081106. The characteristics of the CpG sites selected by LASSO analysis are shown in Tables 2 and 3. The methylation levels of $3 \mathrm{CpG}$ sites were upregulated, and $5 \mathrm{CpG}$ sites were downregulated for miRNA in PAC tissues compared to normal adjacent tissues (Additional file 1: Figure S1). For lncRNA in PAC tissues, the methylation levels of $5 \mathrm{CpG}$ sites were augmented, and those of $2 \mathrm{CpG}$ sites were decreased compared to normal adjacent tissues (Additional file 2: Figure S2). The methylation data of the CpG sites selected by LASSO analysis could clearly discriminate PAC and normal adjacent samples, which was further confirmed by unsupervised hierarchical clustering analyses (Fig. 1c, f).

\section{Predictive and prognostic value of methylation-based classifier}

With the help of the methylation-based classifiers, the risk score of each PAC patient was calculated. And tdROC analysis was adopted to determine the accuracy of the classifiers for predicting the prognosis of PAC. The results obtained from the tdROC curve analysis indicated that these classifiers had favourable predictive and prognostic accuracy at varying follow-up times. For the methylation-based classifier of miRNA, the AUCs were $0.744(95 \% \mathrm{CI} 0.657-0.830)$ at 1 year, 0.869 (95\% CI $0.777-0.961)$ at 3 years, and 0.889 (95\% CI $0.784-0.995$ ) at 5 years (Fig. 2a). For the methylation-based classifier of lncRNA, the AUCs were 0.732 (95\% CI 0.641-0.823) at 1 year, $0.846(95 \%$ CI $0.756-0.936)$ at 3 years and 0.838 (95\% CI 0.707-0.968) at 5 years (Fig. 2d).

Using the median risk score cut-off point, we categorized the PAC patients into two groups, including a high-risk group and a low-risk group. As demonstrated by Kaplan-Meier curves, patients with high-risk scores for the methylation-based classifier of miRNA had worse OS than those who had low-risk scores (HR: 2.42, 95\% CI 1.62-3.63, $\mathrm{P}<0.001$, Fig. $2 \mathrm{~b}$ ). Analogously, the OS of patients with high-risk scores for the methylation-based classifier of lncRNA was shown to be worse than those who had low-risk scores (HR: 2.22, 95\% CI 1.49-3.32,
$\mathrm{P}<0.001$, Fig. 2e). For disease-free survival (DFS) patients, the methylation-based classifier of miRNA or lncRNA could also accurately distinguish OS according to high-risk or low-risk scores (HR: 5.92, 95\% CI 2.1116.62, $\mathrm{P}=0.001$, Fig. 2c; HR: 4.66, 95\% CI 1.54-14.04, $\mathrm{P}=0.002$, Fig. 2f). To further assess the prognostic value of the methylation-based classifiers of miRNA or lncRNA, we stratified patients using clinicopathological risk factors including age and tumour size. Notably, the methylation-based classifier of miRNA (Fig. 3a-d) and LncRNA (Fig. 3e-h) still remained significant for OS.

To compare the prognostic value of methylationbased classifiers and other clinical variables for OS, univariate Cox regression was performed. And the results revealed that tumour grade (HR: $1.56,95 \%$ CI 1.03-2.36, $\mathrm{P}=0.037$ ), tumour size (HR: 2.12 , 95\% CI 1.13-3.98, $\mathrm{P}=0.020$ ), methylation-based classifier of miRNA (HR: 2.56, 95\% CI 1.68-3.90, $\mathrm{P}<0.001)$ and methylationbased classifier of lncRNA (HR: 2.27, 95\% CI 1.51-3.40, $\mathrm{P}<0.001)$ were correlated with OS. Additionally, when the clinal variables and methylation-based classifier of miRNA were adjusted by using multivariable analysis, only our methylation-based classifier of miRNA (HR: 2.78, 95\% CI 1.80-4.27, $\mathrm{P}<0.001$ ) and tumour size (HR: 2.23, 95\% CI 1.52-4.31, $\mathrm{P}=0.017$ ) were identified as potent and independent factors of OS (Table 4). Similarly, the results of multivariate Cox regression models presented that the methylation-based classifier of lncRNA (HR: 2.32, 95\% CI 1.53-3.50, $\mathrm{P}<0.001$ ) and tumour size (HR: 2.07, 95\% CI 1.07-4.00, P =0.032) remained significant independent prognostic factors of OS (Table 5). And tdROC revealed that the methylation-based classifier of miRNA combined with tumour size provided increased accuracy for prediction of OS of $\mathrm{PAC}$ at 1 year $(\mathrm{AUC}=0.686,95 \%$ CI $0.600-0.772)$, 3 years $(\mathrm{AUC}=0.818,95 \% \mathrm{CI} 0.736-0.899)$ and 5 years (AUC $=0.856,95 \%$ CI 0.766-0.946) (Fig. 4a-c), and the methylation-based classifier of lncRNA combined with tumour size also provided a more accurate prediction for OS at 1 year (AUC $=0.677,95 \%$ CI $0.590-0.764$ ) and 3 years (AUC $=0.753,95 \%$ CI 0.641-0.865) (Fig. 4d, e). We were unable to precisely predict 5-year survival rate of PAC patients using the methylation-based classifier of lncRNA combined with tumour size (Fig. 4f).

\section{Identification and functional evaluation of miRNA target genes and genes co-expressed with IncRNA}

There were 8 miRNAs associated with the CpG sites in the methylation-based classifier of miRNA (Table 2), and 7 lncRNAs were correlated with the CpG sites in the methylation-based classifier of lncRNA (Table 3). DIANA microT-CDS was used to identify all of the 2442 predicted miRNA target genes. MEM analysis 

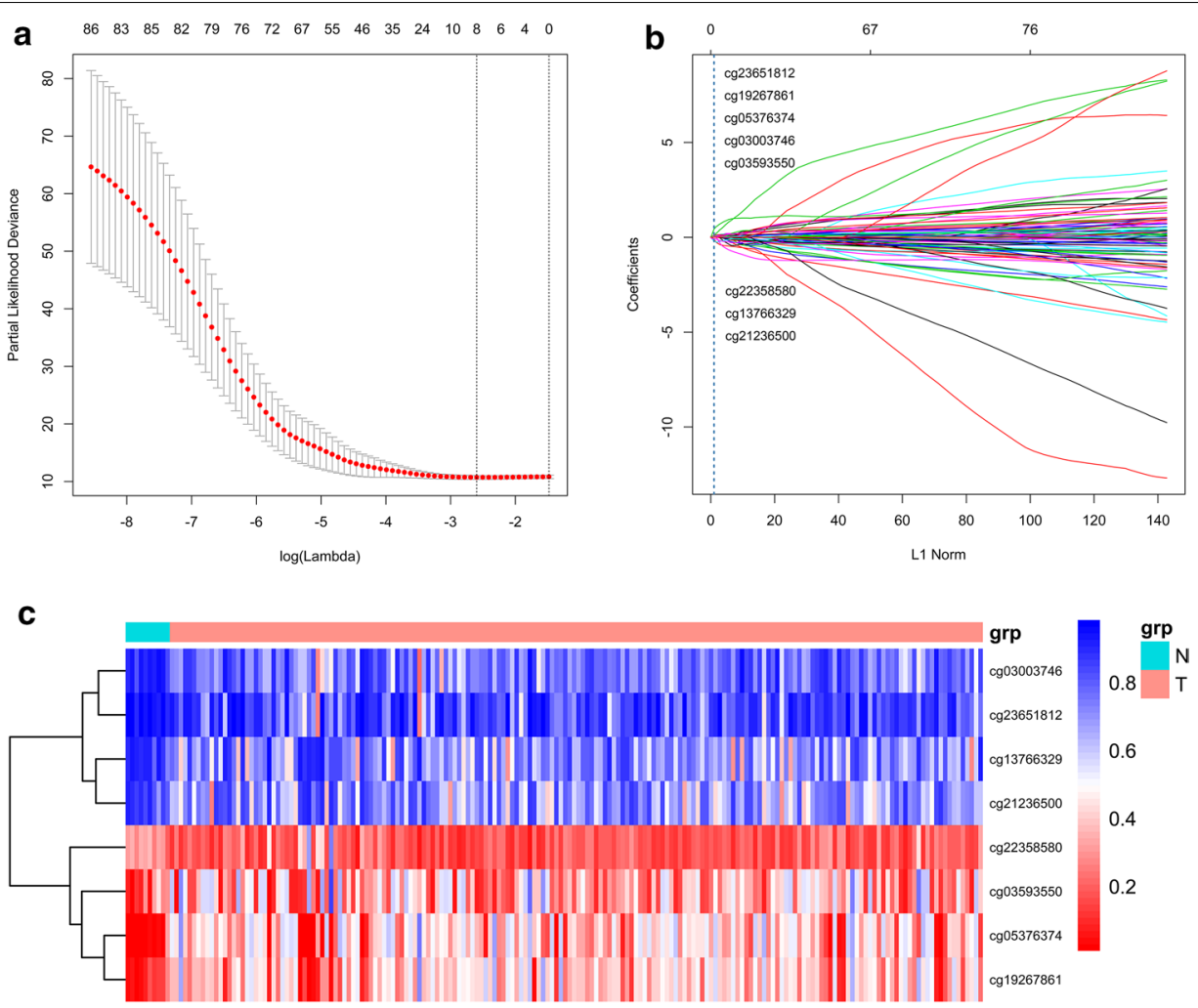

d

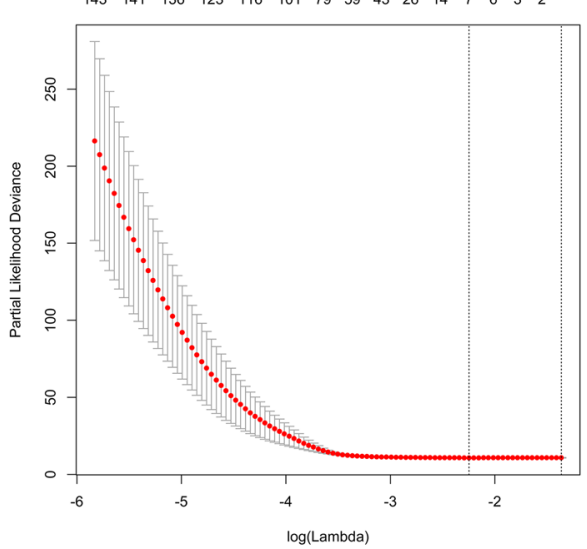

e

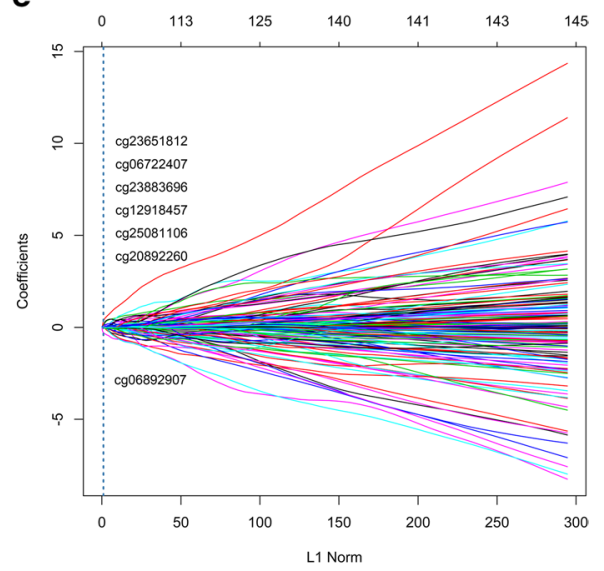

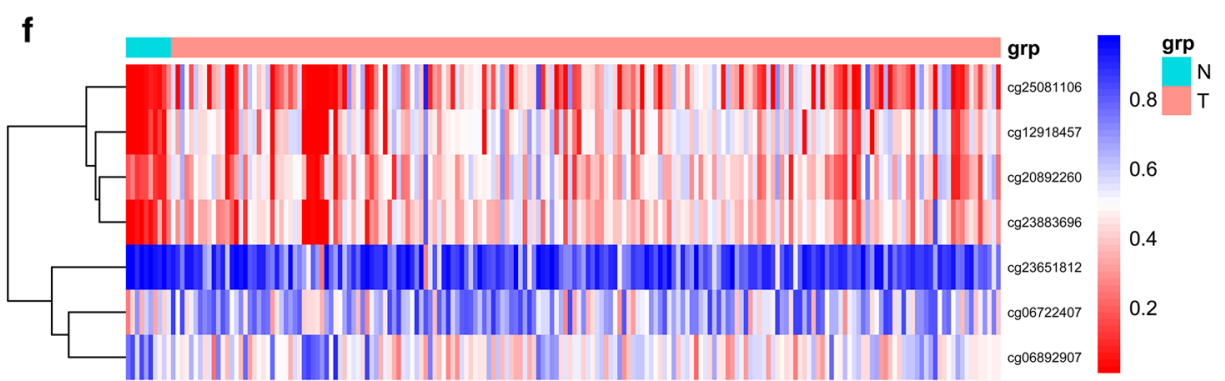

Fig. 1 Construction of the methylation-based classifiers. a-c Methylation-based classifier of miRNA. $\mathbf{d}-\mathbf{f}$ Methylation-based classifier of IncRNA. a, $\mathbf{d}$ Selection CpG sites in LASSO model. $\mathbf{b}$, e LASSO coefficient profiles of CpG sites. $\mathbf{c}$, $\mathbf{f}$ Hierarchical clustering using differentially methylation levels of CpG sites 
Table 2 Characteristics of CpG sites for miRNA selected by LASSO

\begin{tabular}{llllll}
\hline CG_ID & Gene_Symbol & CG_Chromosome_location & Position_to_TSS & CGI_Coordinate & Feature_Type \\
\hline cg03003746 & miR-200a & chr1: $1,167,453-1,167,454$ & TSS1500 & chr1:1,162,663-1,165,204 & S_Shelf \\
cg03593550 & miR-935 & chr19:53,982,150-53,982,151 & TSS200 & chr19:53,982,049-53,982,954 & Island \\
cg05376374 & miR-129-2 & chr11:43,581,370-43,581,371 & TSS200 & chr11:43,580,995-43,581,665 & Island \\
cg13766329 & miR-1249 & chr22: $45,201,099-45,201,100$ & TSS200 & chr22:45,202,840-45,203,199 & N_Shore \\
cg19267861 & miR-124-3 & chr20: $63,178,372-63,178,373$ & TSS200 & chr20:63,174,902-63,179,515 & Island \\
cg21236500 & miR-4479 & chr9: $136,885,445-136,885,446$ & TSS1500 & chr9:136,885,954-136,887,075 & N_Shore \\
cg22358580 & miR-615 & chr12: $54,032,941-54,032,942$ & TSS1500 & chr12:54,033,241-54,034,925 & N_Shore \\
cg23651812 & miR-429 & chr1: 1,168,986-1,168,987 & TSS200 & chr1:1,162,663-1,165,204 & S_Shelf \\
\hline
\end{tabular}

CGI CpG island

Table 3 Characteristics of CpG sites for IncRNA selected by LASSO

\begin{tabular}{|c|c|c|c|c|c|}
\hline CG_ID & Gene_Symbol & CG_Chromosome_location & Position_to_TSS & CGI_Coordinate & Feature_Type \\
\hline cg06722407 & LINC00421 & chr13: 19,344,446-19,344,447 & TSS1500 & chr13:19,344,445-19,345,081 & Island \\
\hline cg06892907 & KB-1732A1.1 & chr8: $102,805,834-102,805,835$ & TSS1500 & chr8:102,806,392-102,807,267 & N_Shore \\
\hline cg12918457 & LINC00900 & chr11: 115,760,030-115,760,031 & TSS1500 & chr11:115,759,680-115,760,399 & Island \\
\hline $\operatorname{cg} 20892260$ & RP11-175E9.1 & chr8: $23,706,512-23,706,513$ & TSS1500 & chr8:23,704,962-23,707,662 & Island \\
\hline $\operatorname{cg} 23651812$ & RP11-465B22.8 & chr1: 1,168,986-1,168,987 & TSS1500 & chr1:1,162,663-1,165,204 & S_Shelf \\
\hline $\operatorname{cg} 23883696$ & RP11-676J15.1 & chr18: $72,867,063-72,867,064$ & TSS1500 & chr18:72,866,730-72,869,636 & Island \\
\hline cg25081106 & MIR4500HG & chr13: 87,671,914-87,671,915 & TSS1500 & chr13:87,671,314-87672,716 & Island \\
\hline
\end{tabular}

CGI CpG island

was performed and identified a total of 2524 genes coexpressed with the lncRNAs. The functional enrichment analysis of those targets and co-expressed genes was performed by DAVID, which revealed significant enriched Gene Oncology (GO) terms and KEGG pathways. For miRNA targets, the GO biological processes were related to regulation of the nucleoplasm (cellular component), transcription (biological process), and protein serine/ threonine kinase activity (molecular function), and the KEGG pathways including the MAPK signalling pathway and the Ras signalling pathway (Fig. 5a, b). For lncRNA co-expressed genes, the GO terms were related to calcium ion transmembrane transport (biological process), cell junction (cellular component), and calcium ion binding (molecular function), and the KEGG pathways including the oxytocin signalling pathway, insulin secretion and calcium signalling pathway (Fig. 5c, d).

\section{Discussion}

PAC is one of the most invasive and lethal carcinomas worldwide, with multiple types of molecular and cellular heterogeneity [14]. Due to limited treatment strategies, the incidence of PAC is increasing yearly and most patients died within 1 year. Therefore, revealing the pathogenic mechanism of PAC will help to advance new treatment strategies. In recent decades, researches have shown that ncRNAs play crucial roles in various cancers with the great improvements in RNA-seq technologies [15]. Although several studies have focused on the functional effect of ncRNA methylation [16], the interrelation between the methylation of ncRNAs and PAC remains still elusive. DNA methylation has been proved to be critical for the regulation of protein-coding gene expressions. Accumulating evidence has illustrated that abnormal methylation at promoter $\mathrm{CpG}$ islands of ncRNAs may be involved in the pathogenesis and prognosis of cancers [11], and this information may contribute to prognostic prediction as well as individualized therapy in these patients.

In this study, we conducted a comprehensive and detailed analysis of the DNA methylation profile of ncRNAs in a cohort of PAC specimens from the TCGA database to investigate the altered DNA methylation patterns in PAC. Furthermore, we identified several important clinically relevant observations. Firstly, according to the 485,577 CpG sites $2 \mathrm{~kb}$ upstream of the TSS in ncRNAs (miRNA or lncRNA), 511 CpG sites of miRNA and 1441 CpG sites of lncRNA were filtered out using different methylation degrees between PAC and the normal neighbouring tissues. The LASSO regression method was used to further determine the ncRNA signature, which identified $8 \mathrm{CpG}$ sites of miRNA and $7 \mathrm{CpG}$ sites of IncRNA with regression coefficients: miR-200a, miR-935, miR-124-3, miR-129-2, miR-1249, miR-4479, miR-615, 

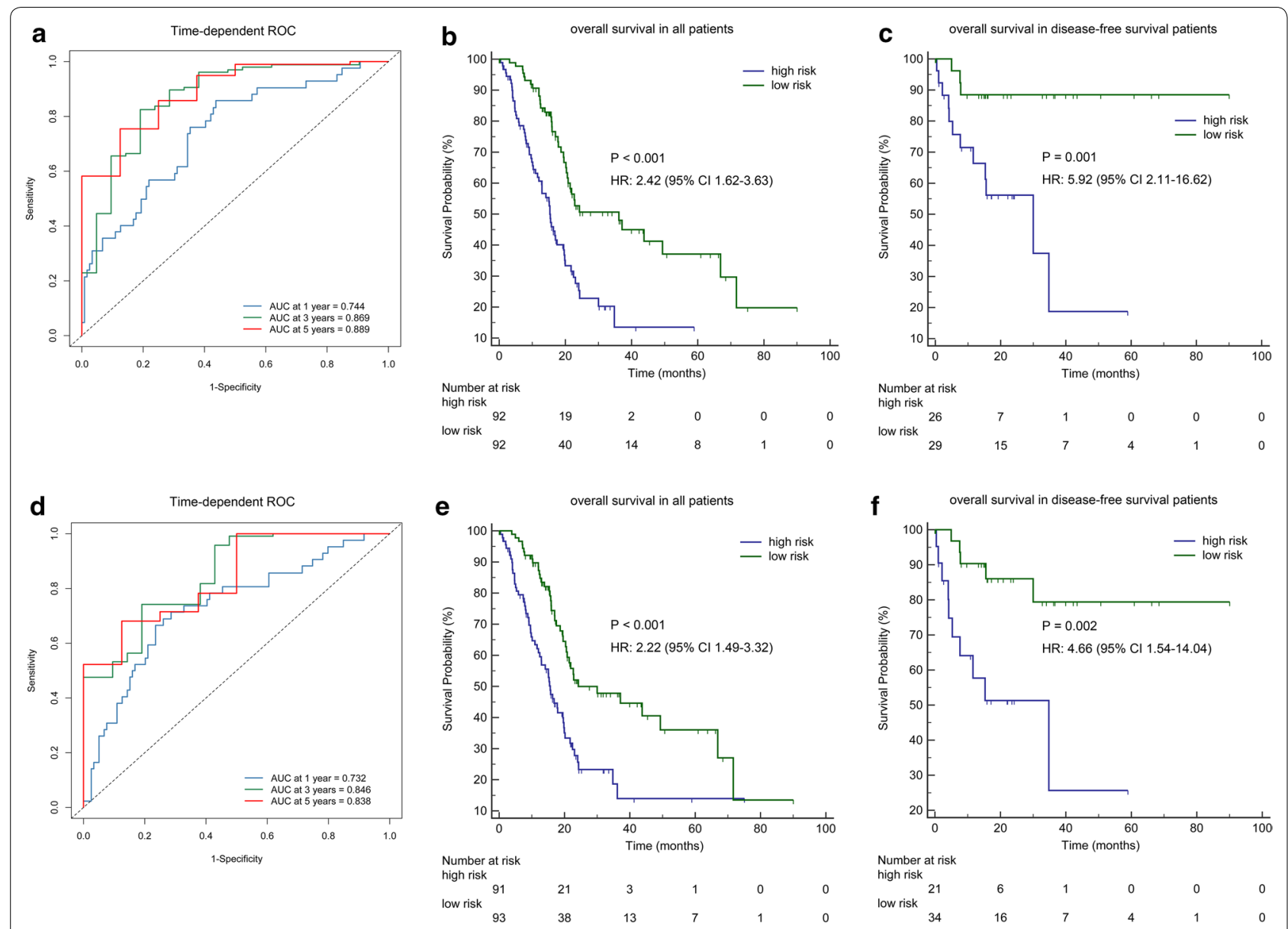

Fig. 2 Time-dependent ROC curves and the survival analysis for the methylation-based classifiers for OS. a-c Methylation-based classifier of miRNA. $\mathbf{d}$-f Methylation-based classifier of IncRNA. a, d Time-dependent ROC curves were applied to assess predictive accuracy for overall survival at varying follow-up times. b, e Kaplan-Meier analysis the overall survival in all patients. c, $\mathbf{f}$ Kaplan-Meier analysis the overall survival in disease-free survival patients. The median of the classifier risk score as a cut-off value to divide the patients into high-risk groups and low-risk groups

miR-429, LINC00421, KB-1732A1.1, LINC00900, RP11-175E9.1, RP11-465B22.8, RP11-676J15.1, and MIR4500HG. Reports have indicated that most of the 8 miRNAs are closely related to tumorigenesis [17-21] while none of the lncRNAs have been studied. Among the 8 miRNAs, miR-200a plays a vital part in the mesenchymal-to-epithelial transition process of pancreatic cancer stem cells by altering cancer migration and invasion [22]. Additionally, miR-935 participates in cell proliferation, migration and apoptosis by reacting with its target, INPP4A, in pancreatic cancer [18]. Emerging evidence illustrated that many ncRNAs are targets of methylationassociated silencing in several diseases, and hypermethylation of the CpG sites could lead to decreased expression of ncRNA [23]. In our study, we confirmed that the methylation levels of $\mathrm{CpG}$ sites were negatively corrected with the expression of miRNA or lncRNA when we screened out the CpG sites of miRNAs and lncRNAs, and this outcome is consistent with other studies. It is reported that promoter hypermethylation of miR-766-3p downregulated the expression of miR-766-3p, indicating the poor prognosis in renal cell carcinoma [24]. Therefore, we speculated that these two methylation-based classifiers of ncRNA can be used to determine the prognosis of pancreatic cancer, possibly by altering the expression of ncRNA to indirectly affect the progression of pancreatic cancer. In addition, the expression pattern of the typical ncRNAs, which were correlated with the CpG sites in the methylation-based classifiers may also could be used for the prognosis of PAC patients. Secondly, the developed methylation-based classifiers of the 8 miRNAs or the 7 lncRNAs can predict the prognosis of PAC precisely, which was the highlight of this study. And tdROC analysis also confirmed the predictive accuracy of the classifiers for OS in PAC patients and in PAC patients with DFS, which is conducive to guiding the implementation of treatment strategies at different stages of the disease. Additionally, the patients can be divided into 

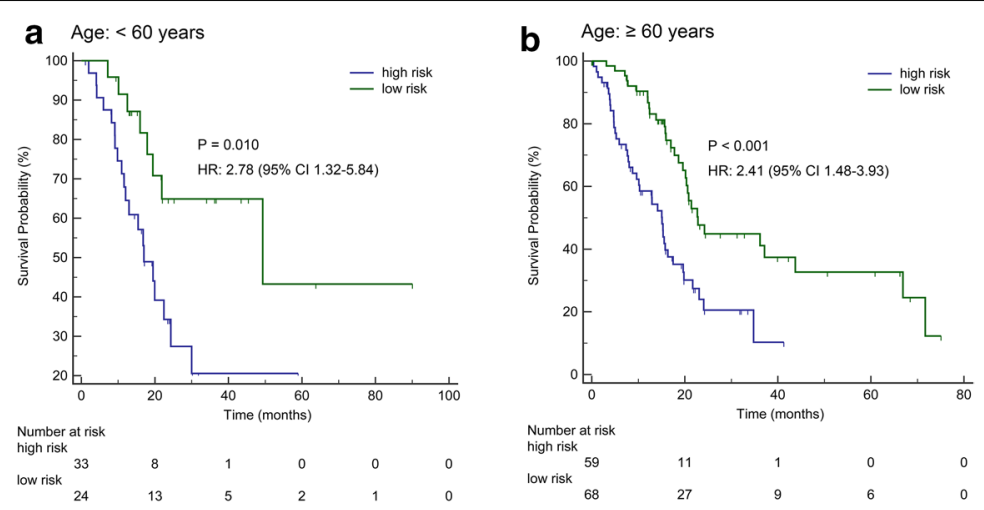

C Pathological stage: low

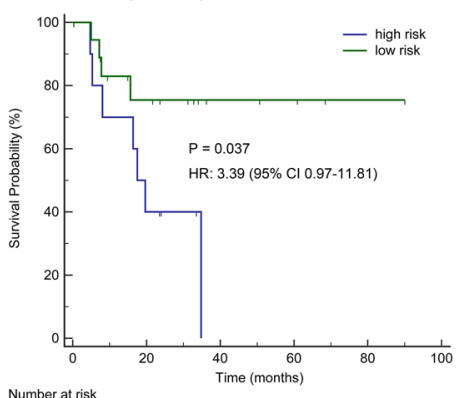

Number at risk

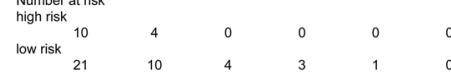

e Age: $<60$ years

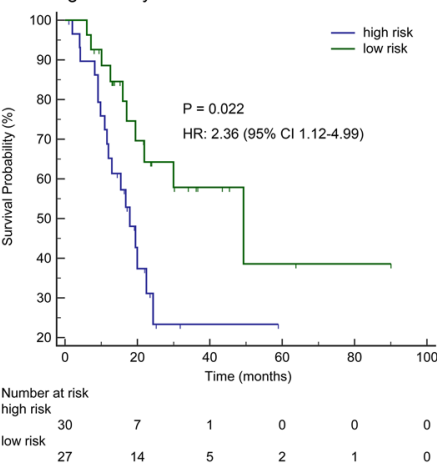

g Pathological stage: low

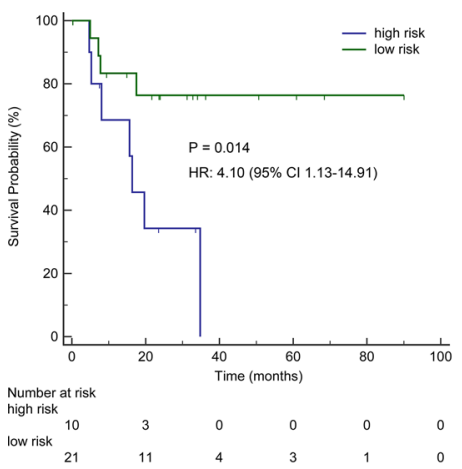

d Pathological stage: high

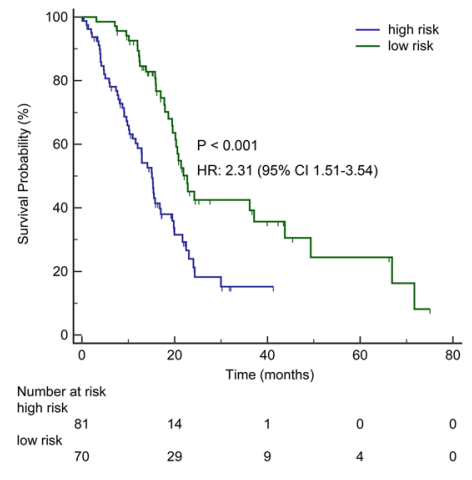

f Age: $\geq 60$ years

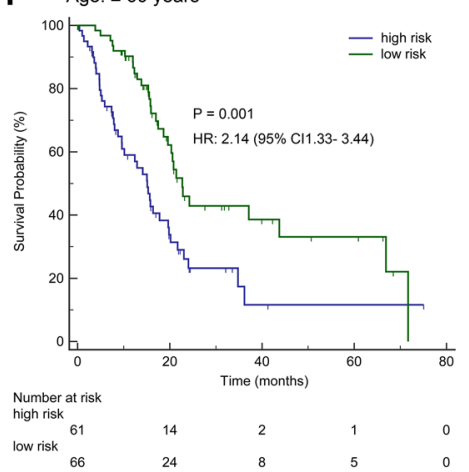

h Pathological stage: high

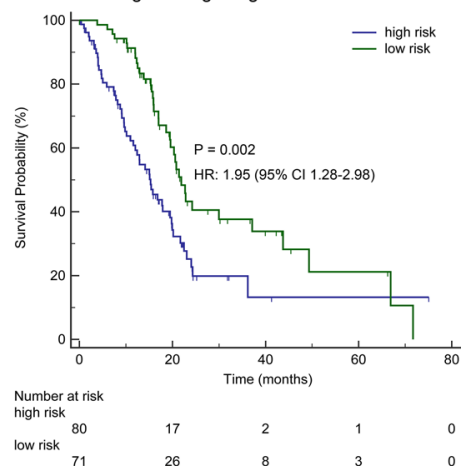

Fig. $\mathbf{3}$ The survival analysis on the basis of the methylation-based classifiers stratified by clinicopathological risk factors. a-d Methylation-based classifier of miRNA. e-h Methylation-based classifier of IncRNA. $\mathbf{a}, \mathbf{b}, \mathbf{e}, \mathbf{f}$ Age. $\mathbf{c}, \mathbf{d}, \mathbf{g}, \mathbf{h}$ Pathological stage. According to the cut-off value, we calculate high and low risk of methylation-based classifier 
Table 4 Univariate and multivariate Cox regression analyses of methylation-based classifier of miRNA for overall survival

\begin{tabular}{|c|c|c|c|c|c|c|}
\hline \multirow[t]{2}{*}{ Prognostic parameter } & \multicolumn{3}{|c|}{ Univariate analysis } & \multicolumn{3}{|c|}{ Multivariate analysis } \\
\hline & HR & $95 \% \mathrm{Cl}$ & $P$ value & HR & $95 \% \mathrm{Cl}$ & $P$ value \\
\hline Age $(\geq 60$ vs. $<60)$ & 1.36 & $0.88-2.11$ & 0.165 & & & \\
\hline Gender (male vs. female) & 0.85 & $0.57-1.26$ & 0.416 & & & \\
\hline $\begin{array}{l}\text { Tumor grade } \\
(\mathrm{G} 3+\mathrm{G} 4 \text { vs. } \mathrm{G} 1+\mathrm{G} 2)\end{array}$ & 1.56 & $1.03-2.36$ & 0.037 & & & \\
\hline $\begin{array}{l}\text { Tumor size } \\
(\mathrm{T} 3+\mathrm{T} 4 \text { vs. } T 1+\mathrm{T} 2)\end{array}$ & 2.12 & $1.13-3.98$ & 0.020 & 2.23 & $1.52-4.31$ & 0.017 \\
\hline $\begin{array}{l}\text { Pathological stage } \\
(I I I+I V \text { vs. I+ II) }\end{array}$ & 1.00 & $0.40-2.46$ & 0.991 & & & \\
\hline $\begin{array}{l}\text { Classifier of miRNA } \\
\text { (high vs. low risk) }\end{array}$ & 2.56 & $1.68-3.90$ & $<0.001$ & 2.78 & $1.80-4.27$ & $<0.001$ \\
\hline
\end{tabular}

Table 5 Univariate and multivariate Cox regression analyses of methylation-based classifier of IncRNA for overall survival

\begin{tabular}{|c|c|c|c|c|c|c|}
\hline \multirow[t]{2}{*}{ Prognostic parameter } & \multicolumn{3}{|c|}{ Univariate analysis } & \multicolumn{3}{|c|}{ Multivariate analysis } \\
\hline & HR & $95 \% \mathrm{Cl}$ & $P$ value & HR & $95 \% \mathrm{Cl}$ & $P$ value \\
\hline Age $(\geq 60$ vs. $<60)$ & 1.36 & $0.88-2.11$ & 0.165 & & & \\
\hline Gender (male vs. female) & 0.85 & $0.57-1.26$ & 0.416 & & & \\
\hline $\begin{array}{l}\text { Tumor grade } \\
(\mathrm{G} 3+\mathrm{G} 4 \mathrm{vs} . \mathrm{G} 1+\mathrm{G} 2)\end{array}$ & 1.56 & $1.03-2.36$ & 0.037 & & & \\
\hline $\begin{array}{l}\text { Tumor size } \\
(\mathrm{T} 3+\mathrm{T} 4 \text { vs. T1 + T2) }\end{array}$ & 2.12 & $1.13-3.98$ & 0.020 & 2.07 & $1.07-4.00$ & 0.032 \\
\hline $\begin{array}{l}\text { Pathological stage } \\
(I I I+I V \text { vs. I+II) }\end{array}$ & 1.00 & $0.40-2.46$ & 0.991 & & & \\
\hline $\begin{array}{l}\text { Classifier of IncRNA } \\
\text { (high vs. low risk) }\end{array}$ & 2.27 & $1.51-3.40$ & $<0.001$ & 2.32 & $1.53-3.50$ & $<0.001$ \\
\hline
\end{tabular}

two groups by this new method based on the median risk score cut-off point. We found that the OS of the patients in the low-risk groups outperformed that of the high-risk group. Consistently, the classifiers could also distinguish difference in OS between the two risk groups according to the clinicopathological risk factors based on the Kaplan-Meier curves, which could help to determine credible individual measures for the patients. Notably, it is urgent to develope additional treatments to improve preoperative risk assessment for PAC patients in lowrisk groups. Meanwhile, the subgroup of PAC patients with high-risk scores identified with these two classifiers might be candidates for more aggressive treatment strategies. There is absolutely no doubt that our miRNA and lncRNA classifiers possessed their own unique prediction compared with the clinicopathological risk factor. And when the two classifiers combination with clinicopathological risk factors, it would provide a more accurate prediction for OS at different times for PAC patients. Surprisingly, we found that the methylation-based classifier of miRNA exhibited better predictive power than that of lncRNA, which is partially due to the indirect effect of lncRNA. Because lncRNA could interact with miRNA as a competitive endogenous RNA and affect mRNA expression [25]. Therefore, the two methylationbased classifier signatures have shown a favourable effect on survival prediction, which will contribute to therapeutic decision-making.

Tumorigenesis is a complex biological process including multifarious epigenetic alterations. Methylation of miRNAs and lncRNAs may participate in the development of carcinoma and play a crucial role in the disease progression. To evaluate the biological function of the ncRNAs in PAC, DAVID performed functional enrichment analysis of the target genes and co-expression genes. And 2442 target genes and 2524 co-expressed genes with abnormal methylation of miRNA and lncRNA, respectively, were identified. The GO results showed the major differences in tumour biology in terms of cellular component, biological process and molecular function. Based on the KEGG pathway analysis results, insulin secretion and the MAPK, Ras, and calcium signalling pathways were significantly enriched, which have previously been validated as vital to the tumorigenesis 

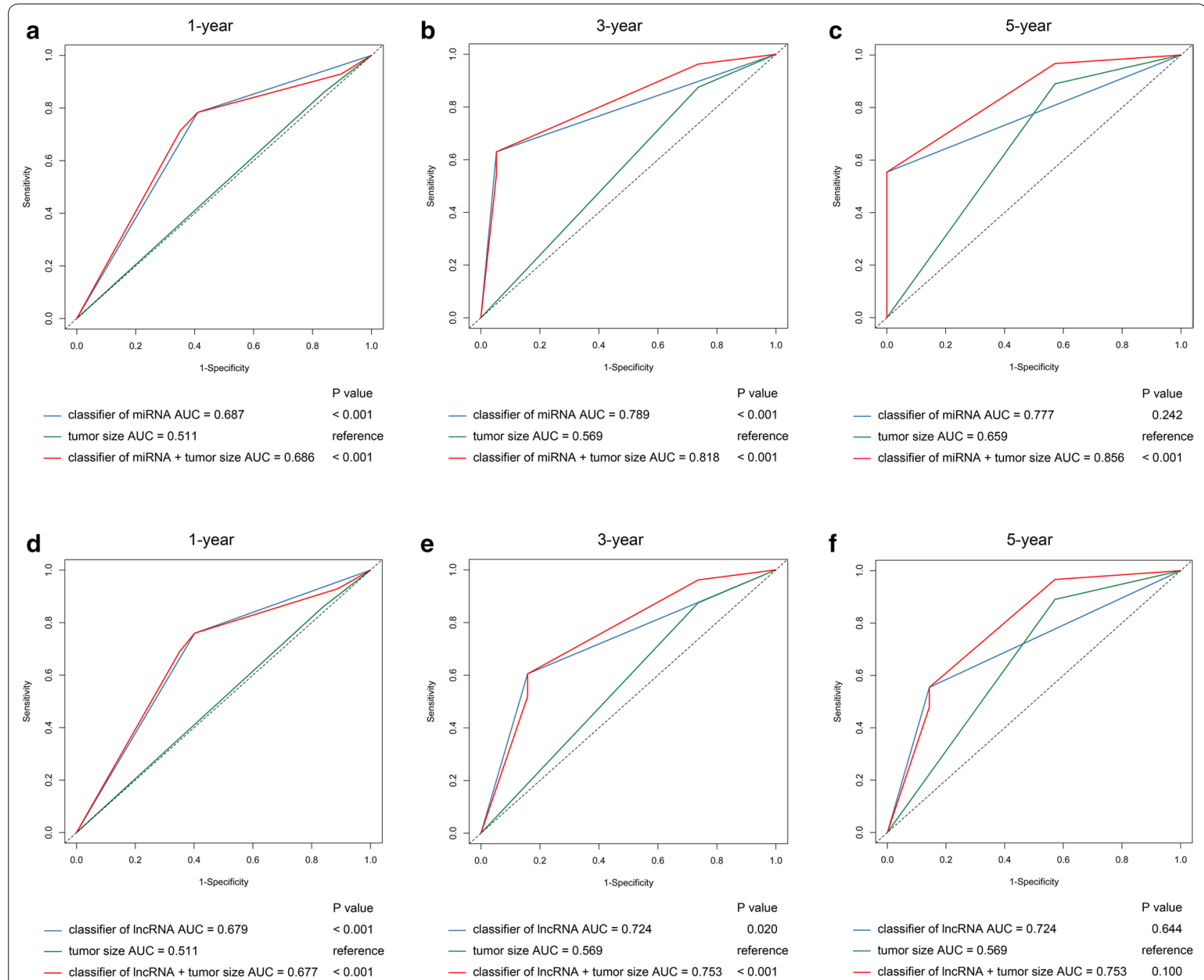

Fig. 4 Time-dependent ROC curves compare the prognostic accuracy of the methylation-based classifiers with clinicopathological risk factors. a-c Methylation-based classifier of miRNA. d-f Methylation-based classifier of IncRNA. a, d 1-year overall survival. b, e 3-year overall survival. c, $\mathbf{f}$ 5-year overall survival

of PAC [26-29]. Notably, the MAPK and Ras signalling pathways are frequently affected in PAC. Yang et al. [30] reported that, in PAC, the tumour suppressor RKIP is regulated by the Ras coded protein, named KRAS, probably via the MAPK-ERK signalling pathway. Moreover, several miRNAs were demonstrated to function as regulators of oncogenic KRAS signalling in PAC [27], which provides novel therapeutic evidence for targeting the Ras signalling pathway in patients. Therefore, these two candidate prognostic classifiers will be beneficial for improving prognosis prediction accuracy and guiding individualized treatment in PAC.

However, there are several limitations in this study. Although we identified aberrantly methylated sites of miRNAs and lncRNAs, whether the alterations represent a cause or an outcome of the cancer process remains unknown. Actually, methylation can occur in both the intergenic regions and repetitive sequences of the human genome and gene promoters. And aberrant hypermethylation of $\mathrm{CpG}$ sites in gene promoters may silence the gene expression that is critical to cell homeostasis, DNA integrity, or genome stability [31]. On the other hand, hypomethylation of $\mathrm{CpG}$ sites in the intergenic regions and repetitive sequences may lead to genomic instability and the loss of gene imprinting [32]. Furthermore, gene expression is related to the degree of methylation [33], which may be discrepant in different genes. Regrettably, this study mainly focused on the association between the methylation level of ncRNA and the prognosis of pancreatic cancer. It will be interesting to study the correlation of these different methylation styles in the future. In addition, no additional experimental data analysis on the 


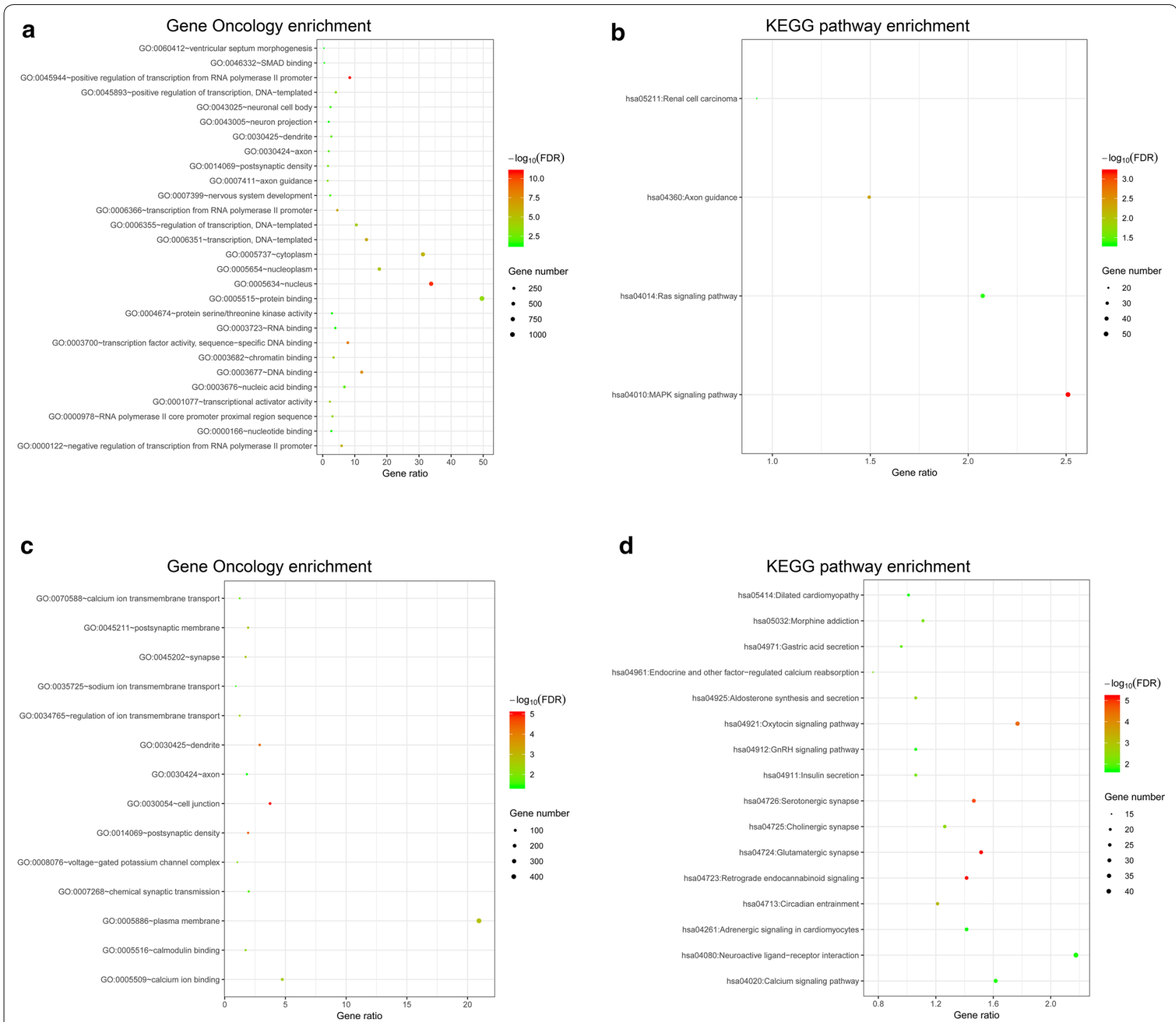

Fig. 5 Functional enrichment analysis of miRNA target genes and IncRNA co-expression genes. a, b miRNA target genes. c, d IncRNA co-expression genes. a, c Gene Oncology (GO) enrichment. b, d KEGG pathway enrichment

underlying mechanisms of miRNAs and lncRNAs was performed in this study. It is urgent to conduct further experimental studies to address these issues.

\section{Conclusion}

In summary, our study identified two methylation-based classifiers of ncRNA associated with OS in PAC. Notably, the altered DNA methylation patterns could accurately predict the prognosis of PAC patients. These patterns are also involved in major cancer signalling pathways known to be crucial in carcinogenesis. Although more experimental studies are required for further confirmation, this is the first study to examine the relationship between aberrant
DNA methylation of ncRNAs and the prognosis of PAC patients, and the results may contribute to the development of individual therapy.

\section{Additional files}

Additional file 1: Figure S1. The difference beta-values of the $8 \mathrm{CpG}$ sites of methylation-based classifier of miRNA between pancreatic adenocarcinoma tissues and normal adjacent tissues.

Additional file 2: Figure S2. The difference beta-values of the $7 \mathrm{CpG}$ sites of methylation-based classifier of IncRNA between pancreatic adenocarcinoma tissues and normal adjacent tissues. 


\begin{abstract}
Abbreviations
ncRNAs: non-coding RNAs; PAC: pancreatic adenocarcinoma; OS: overall survival; miRNAs: microRNAs; IncRNAs: long non-coding RNAs; GCA: gastric cardia adenocarcinoma; TCGA: The Cancer Genome Atlas; LASSO: Least Absolute Shrinkage and Selection Operator; TSS: transcriptional start site; ROC: receiver operating characteristic; AUC: the area under the curve; MEM: MultiExperiment Matrix; FDRs: false discovery rates; DFS: disease-free survival; GO: Gene Oncology.
\end{abstract}

\section{Authors' contributions}

Conceived and designed the study: MTZ. Data collection and analysis: JZ, KQS, WGH, WQW. Generated figures: ZJZ, YYG, TD, XFN. Wrote the manuscript: JZ, YKX. Revised and edited the manuscript: MTZ, CBC. All authors read and approved the final manuscript.

\section{Author details}

${ }^{1}$ Key Laboratory of Diagnosis and Treatment of Severe Hepato-Pancreatic Diseases of Zhejiang Province, The First Affiliated Hospital, Wenzhou Medical University, Wenzhou 325015, Zhejiang Province, People's Republic of China. 2 Precision Medicine Center, The First Affiliated Hospital, Wenzhou Medical University, Wenzhou 325015, Zhejiang Province, People's Republic of China.

\section{Acknowledgements}

We are grateful to all of the reviewers for their comments.

\section{Competing interests}

The authors declare that they have no competing interests.

\section{Availability of data and materials}

The datasets used during the current study are available from the corresponding author on reasonable request.

\section{Consent for publication}

Not applicable.

\section{Ethics approval and consent to participate}

Not applicable.

\section{Funding}

This study was supported by grants from the National Natural Sciences Foundation of China (81570853, 81770630), Science Technology Department of Zhejiang Province, China (2018C03021), Provinces and Ministries Co-Contribution of Zhejiang, China (No.wkj-zj-1706), and Key projects of Wenzhou science and technology bureau (2018ZY008).

\section{Publisher's Note}

Springer Nature remains neutral with regard to jurisdictional claims in published maps and institutional affiliations.

Received: 16 February 2019 Accepted: 13 April 2019

Published online: 23 April 2019

\section{References}

1. Siegel RL, Miller KD, Fedewa SA, Ahnen DJ, Meester RGS, Barzi A, Jemal A. Colorectal cancer statistics, 2017. CA Cancer J Clin. 2017;67(3):177-93.

2. Keane FK, Wo JY, Ferrone CR, Clark JW, Blaszkowsky LS, Allen JN, Kwak EL, Ryan DP, Lillemoe KD, Fernandez-Del Castillo C, et al. Intraoperative radiotherapy in the era of intensive neoadjuvant chemotherapy and chemoradiotherapy for pancreatic adenocarcinoma. Am J Clin Oncol. 2018:41(6):607-12.

3. Jones S, Zhang X, Parsons DW, Lin JC, Leary RJ, Angenendt P, Mankoo $\mathrm{P}$, Carter H, Kamiyama H, Jimeno A, et al. Core signaling pathways in human pancreatic cancers revealed by global genomic analyses. Science. 2008;321(5897):1801-6.

4. Corra F, Agnoletto C, Minotti L, Baldassari F, Volinia S. The network of noncoding RNAs in cancer drug resistance. Front Oncol. 2018;8:327.
5. Djuranovic S, Nahvi A, Green R. miRNA-mediated gene silencing by translational repression followed by mRNA deadenylation and decay. Science. 2012:336(6078):237-40.

6. Li Z, Qiu R, Qiu X, Tian T. SNHG6 promotes tumor growth via repression of P21 in colorectal cancer. Cell Physiol Biochem. 2018;49(2):463-78.

7. Bayraktar R, Ivan C, Bayraktar E, Kanlikilicer P, Kabil NN, Kahraman N, Mokhlis HA, Karakas D, Rodriguez-Aguayo C, Arslan A, et al. Dual suppressive effect of miR-34a on the FOXM1/eEF2-kinase axis regulates triple-negative breast cancer growth and invasion. Clin Cancer Res. 2018;24(17):4225-41.

8. Zou Z, Ma T, He X, Zhou J, Ma H, Xie M, Liu Y, Lu D, Di S, Zhang Z. Long intergenic non-coding RNA 00324 promotes gastric cancer cell proliferation via binding with HuR and stabilizing FAM83B expression. Cell Death Dis. 2018;9(7):717

9. Sato F, Tsuchiya S, Meltzer SJ, Shimizu K. MicroRNAs and epigenetics. FEBS J. 2011;278(10):1598-609.

10. Zhang Z, Dai DQ. MicroRNA-596 acts as a tumor suppressor in gastric cancer and is upregulated by promotor demethylation. World J Gastroenterol. 2019;25(10):1224-37.

11. Guo W, Dong Z, Liu S, Qiao Y, Kuang G, Guo Y, Shen S, Liang J. Promoter hypermethylation-mediated downregulation of miR-770 and its host gene MEG3, a long non-coding RNA, in the development of gastric cardia adenocarcinoma. Mol Carcinog. 2017;56(8):1924-34.

12. Kim SM, Kim Y, Jeong K, Jeong H, Kim J. Logistic LASSO regression for the diagnosis of breast cancer using clinical demographic data and the BI-RADS lexicon for ultrasonography. Ultrasonography. 2018;37(1):36-42.

13. Ge H, Yan Y, Wu D, Huang Y, Tian F. Potential role of LINC00996 in colorectal cancer: a study based on data mining and bioinformatics. Onco Targets Ther. 2018;11:4845-55.

14. Waddell N, Pajic M, Patch AM, Chang DK, Kassahn KS, Bailey P, Johns AL, Miller D, Nones K, Quek K, et al. Whole genomes redefine the mutational landscape of pancreatic cancer. Nature. 2015;518(7540):495-501.

15. Bartonicek N, Maag JL, Dinger ME. Long noncoding RNAs in cancer: mechanisms of action and technological advancements. Mol Cancer. 2016:15(1):43.

16. Wu SC, Kallin EM, Zhang Y. Role of H3K27 methylation in the regulation of IncRNA expression. Cell Res. 2010;20(10):1109-16.

17. Hu B, Qiu-Lan H, Lei RE, Shi C, Jiang HX, Qin SY. Interleukin-9 promotes pancreatic cancer cells proliferation and migration via the miR-200a/ beta-catenin axis. Biomed Res Int. 2017;2017:2831056.

18. Wang C, Feng Z, Jiang K, Zuo X. Upregulation of MicroRNA-935 promotes the malignant behaviors of pancreatic carcinoma PANC-1 cells via targeting inositol polyphosphate 4-phosphatase type I gene (INPP4A). Oncol Res. 2017;25(4):559-69.

19. Fang B, Li G, Xu C, Hui Y, Li G. MicroRNA miR-1249 downregulates adenomatous polyposis coli 2 expression and promotes glioma cells proliferation. Am J Transl Res. 2018;10(5):1324-36.

20. Dong Y, Huo X, Sun R, Liu Z, Huang M, Yang S. IncRNA Gm15290 promotes cell proliferation and invasion in lung cancer through directly interacting with and suppressing the tumor suppressor miR-615-5p. Biosci Rep. 2018;38(5):BSR20181150.

21. Liu D, Song L, Dai Z, Guan H, Kang H, Zhang Y, Yan W, Zhao X, Zhang S. MiR-429 suppresses neurotrophin-3 to alleviate perineural invasion of pancreatic cancer. Biochem Biophys Res Commun. 2018;505(4):1077-83.

22. Lu Y, Lu J, Li X, Zhu H, Fan X, Zhu S, Wang Y, Guo Q, Wang L, Huang Y, et al. MiR-200a inhibits epithelial-mesenchymal transition of pancreatic cancer stem cell. BMC Cancer. 2014;14:85.

23. Suzuki H, Maruyama R, Yamamoto E, Niinuma T, Kai M. Relationship between noncoding RNA dysregulation and epigenetic mechanisms in cancer. Adv Exp Med Biol. 2016;927:109-35.

24. Chen C, Xue S, Zhang J, Chen W, Gong D, Zheng J, Ma J, Xue W, Chen $Y$, Zhai W, et al. DNA-methylation-mediated repression of miR-766-3p promotes cell proliferation via targeting SF2 expression in renal cell carcinoma. Int J Cancer. 2017;141(9):1867-78.

25. Liz J, Esteller M. IncRNAs and microRNAs with a role in cancer development. Biochim Biophys Acta. 2016;1859(1):169-76.

26. Korc M. p38 MAPK in pancreatic cancer: finding a protective needle in the haystack. Clin Cancer Res. 2014;20(23):5866-8.

27. Karmakar S, Kaushik G, Nimmakayala R, Rachagani S, Ponnusamy MP, Batra SK. MicroRNA regulation of K-Ras in pancreatic cancer and opportunities for therapeutic intervention. Semin Cancer Biol. 2017;24:63-71. 
28. Cersosimo E, Pisters PW, Pesola G, McDermott K, Bajorunas D, Brennan MF. Insulin secretion and action in patients with pancreatic cancer. Cancer. 1991;67(2):486-93.

29. Schaal C, Padmanabhan J, Chellappan S. The role of nAChR and calcium signaling in pancreatic cancer initiation and progression. Cancers (Basel). 2015;7(3):1447-71.

30. Yang K, Li Y, Lian G, Lin H, Shang C, Zeng L, Chen S, Li J, Huang C, Huang $K$, et al. KRAS promotes tumor metastasis and chemoresistance by repressing RKIP via the MAPK-ERK pathway in pancreatic cancer. Int J Cancer. 2018;142(11):2323-34.

31. Meng W, Cui W, Zhao L, Chi W, Cao H, Wang B. Aberrant methylation and downregulation of ZNF667-AS1 and ZNF667 promote the malignant progression of laryngeal squamous cell carcinoma. J Biomed Sci. 2019;26(1):13.

32. Bakshi A, Bretz CL, Cain TL, Kim J. Intergenic and intronic DNA hypomethylated regions as putative regulators of imprinted domains. Epigenomics. 2018;10(4):445-61.

33. Krzeminski P, Sarasquete ME, Misiewicz-Krzeminska I, Corral R, Corchete LA, Martin AA, Garcia-Sanz R, San Miguel JF, Gutierrez NC. Insights into epigenetic regulation of microRNA-155 expression in multiple myeloma. Biochim Biophys Acta. 2015;1849(3):353-66.
Ready to submit your research? Choose BMC and benefit from:

- fast, convenient online submission

- thorough peer review by experienced researchers in your field

- rapid publication on acceptance

- support for research data, including large and complex data types

- gold Open Access which fosters wider collaboration and increased citations

- maximum visibility for your research: over $100 \mathrm{M}$ website views per year

At BMC, research is always in progress.

Learn more biomedcentral.com/submissions 